\title{
Effects of Glycyrrhizin in a Mouse Model of Lung Adenocarcinoma
}

\author{
Qing-Ping Deng ${ }^{\mathrm{a}}$ Mao-Jie Wang ${ }^{\mathrm{a}}$ Xing Zeng ${ }^{\mathrm{a}}$ George Gong Chen ${ }^{\mathrm{d}}$ \\ Run-Yue Huang $g^{a, b, c}$
}

aThe Second Affiliated Hospital, Guangzhou University of Chinese Medicine (Guangdong Provincial Hospital of Chinese Medicine), Guangzhou, China; bUniversity Medical Center Utrecht, Utrecht, The Netherlands; 'Guangdong Provincial Key Laboratory of Clinical Research on Traditional Chinese Medicine Syndrome, Guangzhou, China; 'Department of Surgery, The Chinese University of Hong Kong, Shatin, N.T., Hong Kong

\section{Key Words}

Glycyrrhizin • Cisplatin • Thromboxane A2 - Lung adenocarcinoma • Animal model

\begin{abstract}
Background: Currently, there is a global attempt to identify potential anti-cancer agents with low toxicity. Previous studies have found that glycyrrhizin exerts anti-cancer action with low toxicity through suppressing thromboxane A2 (TxA2) in lung cancer cell lines. However, these effects have not yet been determined in animal models of lung cancer. Methods: Human lung adenocarcinoma xenografts were established in nude mice by the introduction of A549 cells with stable transfection of the TxA2 receptor (TP $\alpha)$. The animal model was confirmed by the hematoxylin and eosin (H\&E) method. Tumor-bearing mice were then administered graded concentrations of glycyrrhizin, cisplatin or both. After the treatments, body weights of all animals were recorded, and immunohistochemistry staining of lung tissues and serum biochemistry detection of aspartate amino transferase (AST), alanine amino transferase (ALT), urea and creatinine were carried out. Results: Treatment with glycyrrhizin alone or the combination of cisplatin and glycyrrhizin profoundly reduced expression of thromboxane synthase (TxAS) as well as proliferating cell nuclear antigen (PCNA), recovered the body weight, and rescued damage of liver and kidney in tumor-bearing mice. Although it inhibited PCNA expression, cisplatin could not significantly suppress TxAS expression. Because of a positive feedback loop between TP $\alpha$ and TxAS, the effects of glycyrrhizin are possibly attributable to the suppression of the TXA2 pathway. Conclusions: This study provides in vivo evidence to support glycyrrhizin as a potential candidate for developing new regimens to overcome tumor progression and the resistance and toxicity of cisplatin.

\section{Introduction}

Lung adenocarcinoma is a major subtype of lung cancer, with a $15 \%$ overall 5 -year survival rate [1]. There is a global attempt to design more powerful drugs for the treatment

Prof. Run-Yue Huang

and Prof. Xing Zeng

\section{KARGER}

Dept Rheumatol and Central Lab, The Second Affiliated Hospital, Guangzhou University of Chinese Medicine (Guangdong Provincial Hospital of Chinese Medicine), Guangzhou 510006 (China), Nowadays visiting at University Medical Center Utrecht, (The Netherlands); and The Second Affiliated Hospital, Guangzhou University of Chinese Medicine (Guangdong Provincial Hospital of Chinese Medicine), Guangzhou 510006 (China)

E-Mail ry_huang@hotmail.com / zengxing-china@163.com 
of lung cancer with low toxicity, but the results are unsatisfactory. Fortunately, emerging evidence has demonstrated that some herbal drugs, such as the active components of licorice, could be better choices to improve the current therapeutic strategy [2-4].

Previous studies have shown that glycyrrhizin inhibits proliferation and induces apoptosis in in vitro models of lung cancer $[1,3,5]$. As an inhibitor of high-mobility group box 1 (HMGB1), glycyrrhizin may induce TxAS through activating toll-like receptor 4 (TLR4) and the subsequent signaling such as nuclear factor (NF)- $\kappa B[6,7]$. The anti-cancer effects of glycyrrhizin can be partly due to the suppression of thromboxane synthase (TxAS) activity, which is reflected by the production of thromboxane A2 (TxA2) [1, 3].

In the last decades, TxA2 has been well established as a tumor promoter in many types of cancers [8-16]. In particular, TxAS and the $\alpha$ isoform of the TxA2 receptor (TP $\alpha$ ) can facilitate lung tumor growth and metastasis $[10,16]$, and intriguingly, there is a positive feedback loop between TxAS and TP $\alpha$ in lung tumors [11]. Briefly, following activation of TP $\alpha$ by specific binding with TxA2, several transcription factors, such as nuclear factor (NF)- $\mathrm{BB}$ and cyclic adenosine monophosphate response element binding protein (CREB), are subsequently activated to induce expression of TxAS, which is the key enzyme for the biosynthesis of TxA2 [8-11, 17]. In addition to being a tumor promoter, TxA2 possibly can be used as a biomarker for tumor cachexia [6] because TxA2 is believed to be involved in the process of cancer cachexia through inducing the chronic inflammatory process [4]. Moreover, TxA2 has been implicated in resistance to chemotherapy. For instance, there is a report showing that endogenous mesenchymal stem cells can induce resistance to chemotherapy in cancer, while the effect can be prevented by blocking TxAS [6]. Therefore, by blocking the TxA2 related pathway, it is possible to reverse cachexia and overcome cisplatin resistance in lung cancer.

Cisplatin is part of the standard of care for patients with lung cancer. However, resistance in some patients and the toxicity of this drug are still major problems that need to be carefully considered. It is known that the cytotoxic effect of cisplatin is mediated in part through the TxA2 pathway $[15,18]$, and interestingly, glycyrrhizin has been demonstrated to reverse cisplatin resistance in hepatocellular carcinoma cells [3]. Therefore, based on previous in vitro studies $[4,6,12]$, the herbal drug glycyrrhizin can possibly be used as an alternative medication to treat cancer patients. Furthermore, there may be some benefits when combining cisplatin with glycyrrhizin, for example, low or minimal toxicity. Considering that there are no in vivo models to test this possibility, this study was designed to further determine the anti-cancer action of glycyrrhizin and the effects of combined treatment with glycyrrhizin and cisplatin in a mouse model of lung adenocarcinoma.

\section{Materials and Methods}

\section{Plasmid Construction and Stable Transfection}

The construction procedure is shown in Fig. 1A. Double restriction enzyme digestion was applied to the recovered target gene fragment and eukaryotic expression vector pcDNA3.1. Under the guidance of the T4 DNA ligase system instructions, the purified target fragment of the TP $\alpha$ gene was directionally ligated into the pcDNA3.1 vector, and the ligation was transformed subsequently. After amplification, the resulting recombinant eukaryotic expression vector was designated as pcDNA3.1-TP $\alpha$. pcDNA3.1-TP $\alpha$ was digested using BamHI and XhoI and then was evaluated by agarose gel electrophoresis. The recombinant plasmid was further sequenced to confirm its sequence by GENEWIZ Company (Suzhou, China).

Stable transfection of A549 cells with the pcDNA3.1-TP $\alpha$ plasmid was carried out using Lipofectamine 2000 (Invitrogen, CA, USA) and G418 (Thermo Fisher Scientific, MA, USA) selection [16]. The stably transfected cells were named A549-TP $\alpha$ cells.

\section{Animal models and Treatments}

All of the procedures for handling of animals abided by the guidelines of Guangzhou University of Chinese Medicine for the care and use of laboratory animals and were approved by the institutional animal research ethical committee. 


\section{Cellular Physiology Cell Physiol Biochem 2017;41:1383-1392

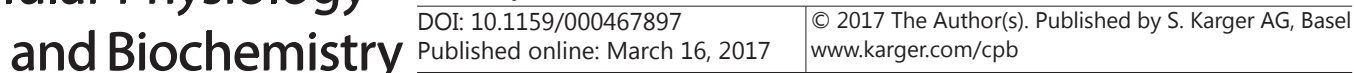 \\ Deng et al.: Glycyrrhizin Effects in a Lung Adenocarcinoma Model}

For tumor formation in nude mice, $1 \times 10^{7}$ monodispersed A549-TP $\alpha$ cells were suspended in $200 \mu \mathrm{l}$ of serum-free RPMI 1640 medium and were injected into the tail vein of 6-week-old female nude mice. Histopathological examination was performed using hematoxylin and eosin (H\&E) staining, as described previously [1].

Four weeks after injection, animals were treated with graded concentrations of glycyrrhizin, cisplatin, or cisplatin combined with glycyrrhizin for 30 days. After treatment, the mice were sacrificed by an excess dose of anesthetic ether and cervical dislocation. Whole blood was collected from the orbital sinus and was subsequently centrifuged to yield serum. The lungs were excised and stored immediately in a liquid nitrogen tank until the experiments were performed.

Glycyrrhizin (>98\% purification) and cisplatin were purchased from ALADDIN Chemical Co., Ltd. (Shanghai, China).

\section{Experimental design}

The design of this study is shown in Fig. 1B, and it was enlightened by our previous in vivo experiments [1]. Sixty nude mice were grouped into 9 groups. Nine mice with no xenografts and treatments served as the normal controls. Four weeks after inoculation with A549-TP $\alpha$ cells, 3 mice were randomly selected from the modeling group and Normal control group for pathological examination using H\&E staining, as shown in Fig. 1C, to confirm the successful establishment of the xenograft animal model of lung adenocarcinoma. Six xenograft mice served as model controls, and the other tumor-bearing mice were treated for 30 days.

For the treatments, the xenograft mice were randomly assigned into the other 7 groups (Fig. 1B): glycyrrhizin treatment with $15 \mathrm{mg} / \mathrm{kg} / \mathrm{d}$ (Low GL), glycyrrhizin treatment with $45 \mathrm{mg} / \mathrm{kg} / \mathrm{d}$ (Middle GL), glycyrrhizin treatment with $135 \mathrm{mg} / \mathrm{kg} / \mathrm{d}$ (High GL), cisplatin treatment only, combination treatment of cisplatin with Low GL, combination treatment of cisplatin with Middle GL, and combination treatment of cisplatin with High GL. The concentration of cisplatin for administration was $2.5 \mathrm{mg} / \mathrm{kg} / \mathrm{d}$. The concentrations of glycyrrhizin and cisplatin used in this study were according to those in previous reports $[1,19,20]$. Each group had 6 mice. After 30 days of treatments, the body weight of all mice was recorded, and the samples were collected to conduct the following experiments.

\section{Immunohistochemistry and serum biochemistry studies}

The lung tissues were sectioned, and immunohistochemistry (IHC) staining was conducted using the anti-TxAS antibody (1:100; Abcam, Cambridge, UK) or anti-PCNA (1:200; Santa Cruz, CA, USA), as previously reported [17]. The immunohistochemical images were quantified using Image-Pro Plus 6.0 software (Media Cybernetics, MD, USA).

The sera were processed for biochemical studies. Analytical kits for detection of aspartate amino transferase (AST), alanine amino transferase (ALT), urea and creatinine were purchased from DIAN diagnostics (Shanghai, China).

Statistical analyses

Data generated from at least three independent experiments were subjected where appropriate to Student's t-test or one-way ANOVA followed by Bonferroni correction. Statistical significance was considered as a two-side p-value of $<0.05$ using SPSS 21.0 (SPSS, Chicago, IL). Data for the experiment group means \pm SD were graphed using Microsoft Excel (Microsoft Corporation, WA, USA).

\section{Results}

Establishment of the xenograft mouse model

We used the method addressed in our previous report to establish the nude mouse model of lung adenocarcinoma [1]. Pathological examination confirmed the successful establishment of the xenograft animal model, as shown in Figs. $1 \mathrm{C}$ and D. In lung tissues, the irregular arrangement of cancer cells and irregular shape of the nucleus are shown, and the phase of cell division is visible (Fig. 1D). 


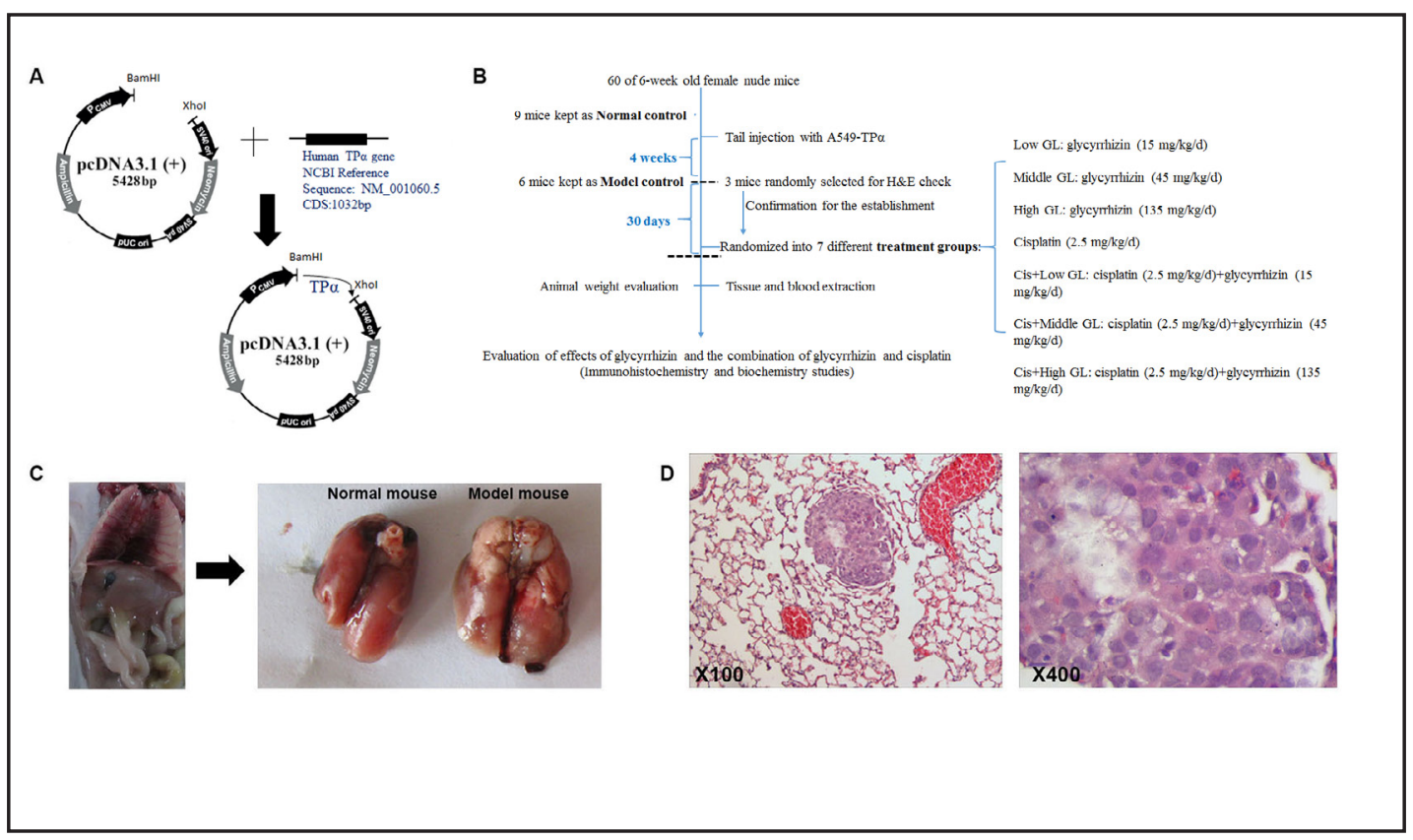

Fig. 1. A, Schematic representation of the construction of the pcDNA3-TP $\alpha$ plasmid. B, Experimental design. $\mathrm{C}$, Three mice in the Normal control group and 3 mice in the Model control group were randomly selected and dissected to determine whether lung adenocarcinoma was established after 4 weeks of A549-TP $\alpha$ cell inoculation. D, Pathological examination of lung adenocarcinoma using H\&E staining. The left panel shows low power $(\times 100)$, and the right panel shows high power $(\times 400)$. Blue, nuclear staining; red, erythrocytes in blood vessels.

\section{Effects of glycyrrhizin and cisplatin on TxAS and PCNA expression}

Protein expression of TxAS and PCNA in lung tissues obtained from mice was detected by IHC staining, and the representative images are shown in Fig. 2 and Fig. 3, respectively. Compared with lung tissues obtained from normal controls, both TxAS and PCNA were overexpressed in lung specimens from tumor-bearing mice (model control). Quantification of IHC staining showed that expression levels of TxAS and PCNA in the model control were $(187.6 \pm 9.1) \%$ and $(239.1 \pm 47.1) \%$ of the normal control, respectively (Fig. 4), further confirming lung tumor formation in animals.

Unsurprisingly, glycyrrhizin suppressed the protein expression of both TxAS and PCNA. Treatment with cisplatin decreased PCNA expression but could not significantly suppress TxAS expression (Figs. 2 and 3). Quantitative data in Fig. 4 show that treatment with 15 $\mathrm{mg} / \mathrm{kg} / \mathrm{d}, 45 \mathrm{mg} / \mathrm{kg} / \mathrm{d}$, and $135 \mathrm{mg} / \mathrm{kg} / \mathrm{d}$ glycyrrhizin reduced the protein level of TxAS by approximately $30.6 \%, 50.2 \%$, and $72.5 \%$ of the model control, respectively, and also suppressed the PCNA level by approximately $40.4 \%, 86.6 \%$, and $106.6 \%$ of the model control.

As illustrated by Fig. 4A, cisplatin treatment suppressed the TxAS level by $24.5 \%$ of the model controls ( $p>0.05$ ), but the level is still almost $163.2 \%$ of the normal control ( $p<$ 0.01). The addition of glycyrrhizin to some extent enhanced the inhibitory effect of cisplatin on TxAS expression (Fig. 2). For example, as seen in Fig. 4A, the combination of $15 \mathrm{mg} / \mathrm{kg} / \mathrm{d}$ glycyrrhizin with $2.5 \mathrm{mg} / \mathrm{kg} / \mathrm{d}$ cisplatin reduced the protein expression of TxAS in lung tissue of tumor-bearing mouse to $146.3 \%$ of the normal control ( $p<0.05)$, which is also much lower than treatment with $15 \mathrm{mg} / \mathrm{kg} / \mathrm{d}$ glycyrrhizin alone $(157.1 \%$ of normal control, $\mathrm{p}<$ 0.05). Consistent with IHC staining, quantification data presented that cisplatin profoundly inhibited PCNA expression, and this effect was promoted by the additional administration of glycyrrhizin (Figs. 3 and 4B). The effect of suppressing PCNA and TxAS was similar between $135 \mathrm{mg} / \mathrm{kg} / \mathrm{d}$ glycyrrhizin treatment alone and the combination of $2.5 \mathrm{mg} / \mathrm{kg} / \mathrm{d}$ cisplatin and $135 \mathrm{mg} / \mathrm{kg} / \mathrm{d}$ glycyrrhizin (Fig. 4). These results indicate that combined treatment with 
Fig. 2. Representative photomicrographs of IHC detection of TxAS in lung tissues. TxAS protein was predominantly expressed in the cytoplasm of lung cells. Brown, TxAS staining; blue, nuclear staining.

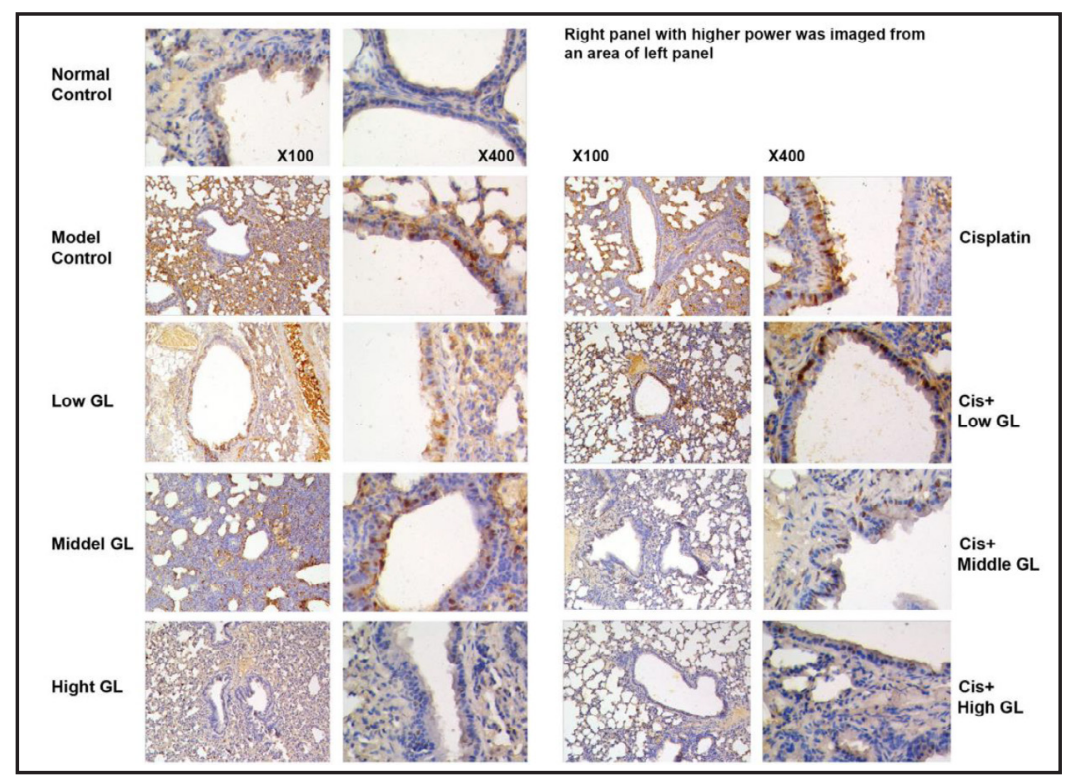

Fig. 3. Representative photomicrographs of IHC detection of PCNA in lung tissues. PCNA protein was mainly shown in nuclei. Brown, PCNA staining; blue, nuclear staining.

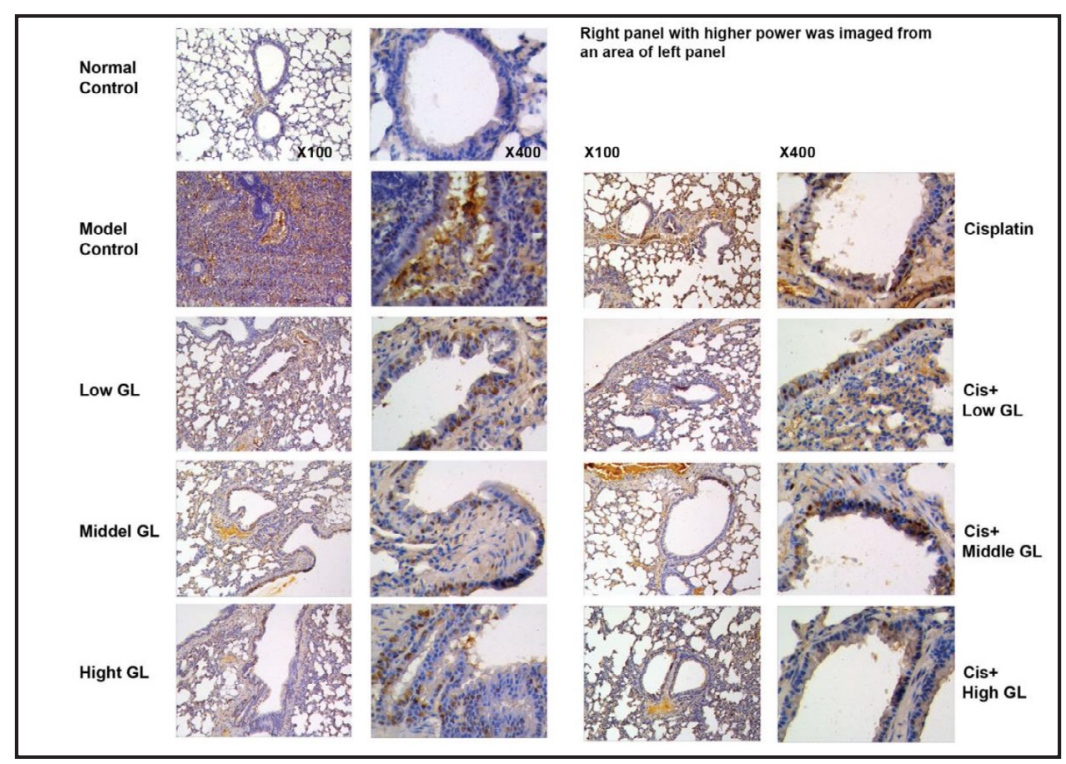

cisplatin and glycyrrhizin can reduce the clinical dosage of cisplatin, thereby decreasing the unpredictable side effects without reducing the therapeutic efficacy.

Glycyrrhizin reverses the loss of body weight caused by tumor bearing

It is well known that cancer cachexia is manifested by loss of body weight. As shown in Fig. $5 \mathrm{~A}$, the body weight of mice in the model control was almost $86.5 \%$ of that of normal mice, suggesting that cancer cachexia had progressed in tumor-bearing animals. Treatment with glycyrrhizin led to a significant improvement in weight loss in tumor mice. In particular, administration of $135 \mathrm{mg} / \mathrm{kg} / \mathrm{d}$ glycyrrhizin alone or combined with cisplatin almost recovered the body weight lost in tumor-bearing mice (Fig. 5A). These data suggest that glycyrrhizin attenuated cachexia progression; therefore, the combination of glycyrrhizin with cisplatin may be more beneficial than chemotherapy alone.

Glycyrrhizin protects the liver and kidney function from tumor bearing and cisplatin treatment

Liver and kidney function was determined by evaluating the serum levels of AST, ALT, urea, and creatinine. As shown in Figs. $5 \mathrm{~B}$ and $\mathrm{C}$, tumor bearing induced the serum levels KARGER 


\section{Cellular Physiology Cell Physiol Biochem 2017;41:1383-1392

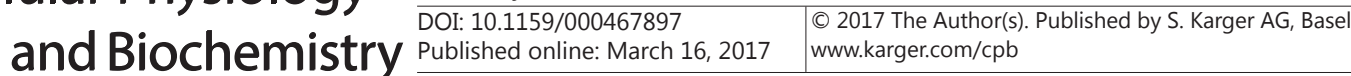 \\ Deng et al.: Glycyrrhizin Effects in a Lung Adenocarcinoma Model}

Fig. 4. Quantification of IHC staining of TxAS and PCNA by Image-Pro Plus 6.0. Six areas were randomly selected from each section and were automatically quantified by Image-Pro Plus 6.0 software, and the integrated optical density (IOD) was evaluated. The results are presented as percentages of the controls. ${ }^{*} \mathrm{p}<0.05$ and $* * \mathrm{p}<0.01$, compared with the normal control; ${ }^{\wedge} \mathrm{p}<0.05$ and $\wedge \wedge \mathrm{p}<0.01$, compared with the model control; $\Delta$ $\mathrm{p}<0.01$, compared with cisplatin treatment.

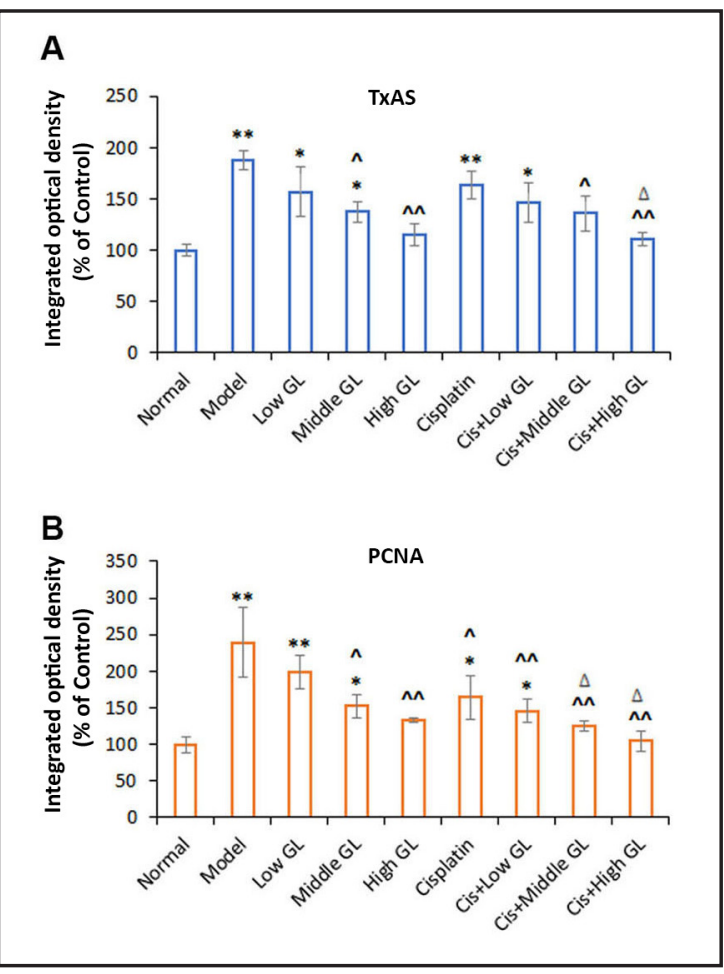

of AST and ALT to approximately 1.2- and 1.9-fold of the normal control, respectively, and enhanced the serum levels of urea and creatinine to 1.4- and 1.3-fold of the normal controls. Because it is well known that cisplatin has multi-organ toxicity, it is unsurprising that treatment with cisplatin did not relieve the liver and kidney damage by tumor bearing and even slightly increased the urea level. Strikingly, administration with graded concentrations of glycyrrhizin profoundly improved the function of the liver and kidney in tumor-bearing mice. Collectively, the high levels of serum AST, ALT, urea and creatinine induced by tumor bearing could be reversed by glycyrrhizin alone or the combination of glycyrrhizin and cisplatin, suggesting the protective effect of this herbal compound on animals with tumors or toxicity of chemotherapy drugs.

\section{Discussion}

The present study provided in vivo evidence to further support the anti-tumor effects of glycyrrhizin and demonstrated that this herbal compound holds a protective effect to somewhat stop cachexia progression and damage to important organs. In addition, because drug combinations have been used as the standard chemotherapeutic strategy in lung cancer [21], the combination of cisplatin and glycyrrhizin has the potential to be developed as a regimen in clinical practice with many benefits.

Two lung adenocarcinoma cell lines, A549 and NCI-H23, which have been widely used in cancer research, were detected without any transfection in our previous reports $[1,11]$. It was found that both TP $\alpha$ and TxAS are abundant in A549 but limited in NCI-H23 cells. In addition, between these two cell lines, only A549 cell growth was significantly suppressed by glycyrrhizin in a dose-dependent manner. Therefore, previous studies have suggested that glycyrrhizin suppressed lung adenocarcinoma cell growth to some extent by blocking the TxA2-related pathway. Importantly, we established a mouse model of lung adenocarcinoma previously, and we demonstrated that glycyrrhizin has an anti-tumor effect with suppression of TxA2 biosynthesis in this model [1]. In this study, the same method was used to establish the animal model first. We stably transfected A549 cells with TP $\alpha$ for the three following 


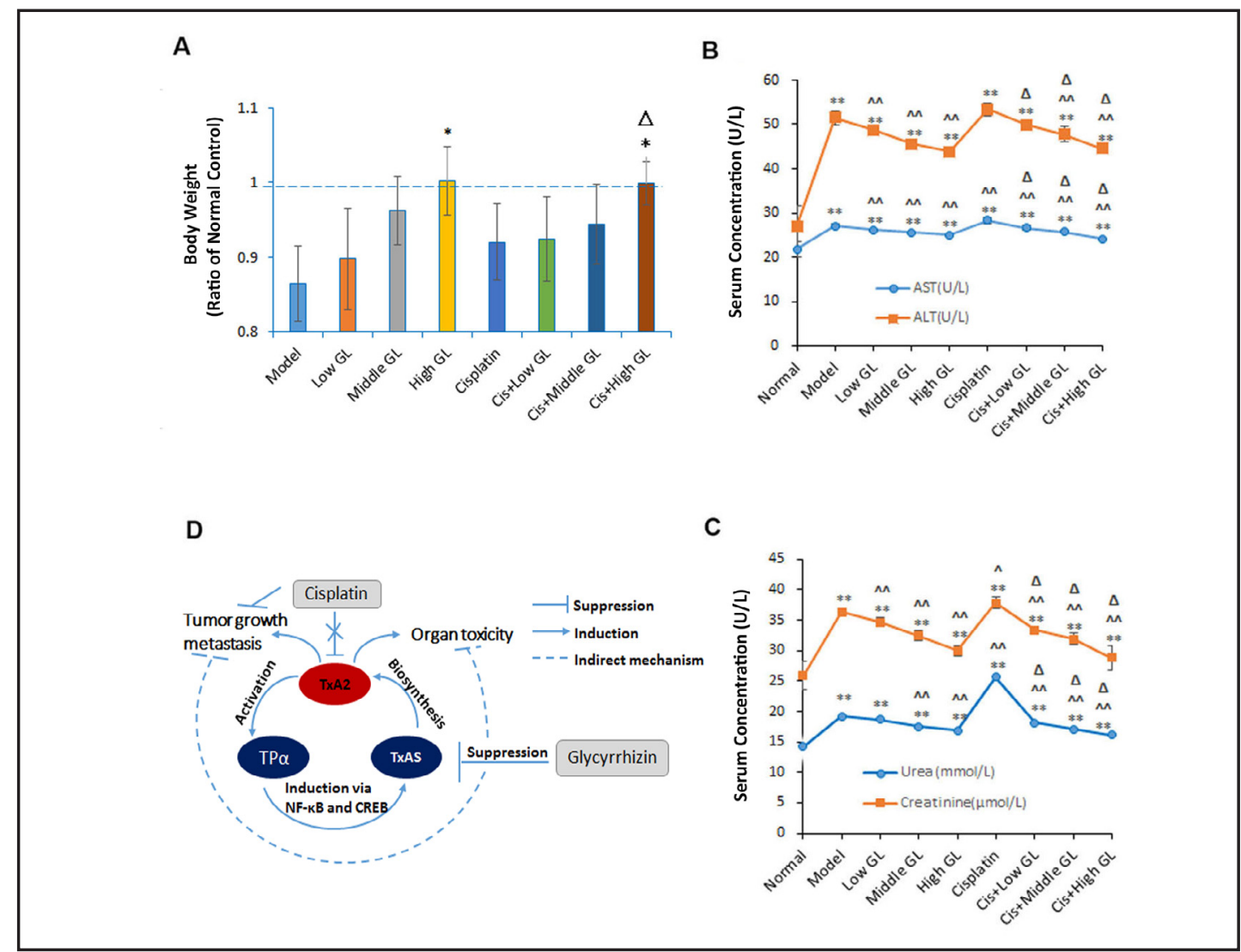

Fig. 5. A, Evaluation of the body weight of animals. The data are expressed as means \pm SD and presented as the ratio of normal controls. The dotted line of 1 represents normal controls. ${ }^{*} p<0.05$, compared with the model control; $\Delta \mathrm{p}<0.05$, relative to cisplatin treatment. B and C, Detection of the serum levels of AST, ALT, urea and creatinine. The data are expressed as means \pm SD of three independent experiments in triplicate. * $\mathrm{p}<0.05$ and ${ }^{* *} \mathrm{p}<0.01$, compared with the normal control; ${ }^{\wedge} \mathrm{p}<0.05$ and ${ }^{\wedge} \wedge \mathrm{p}<0.01$, compared with the model control; $\Delta \mathrm{p}<0.01$, compared with cisplatin treatment. D, Proposed mechanism of glycyrrhizin action in lung adenocarcinoma. Through activation of the transcription factors NF- $\kappa$ B and CREB, an auto-regulatory feedback loop between TP $\alpha$ and TxAS is induced. Cisplatin shows a lack of inhibition of TxA2 production, and the multiple organ toxicity of cisplatin is somewhat related to the TxA2 pathway. By suppressing TxAS, glycyrrhizin not only exerts a synergistic effect with cisplatin on tumor progression but also lowers the toxic action of cisplatin in lung adenocarcinoma. Abbreviations: TxA2, thromboxane A2; TxAS, thromboxane synthase; $\mathrm{TP} \alpha, \alpha$ isoform of thromboxane receptor; NF- $\kappa \mathrm{B}$, nuclear factor $\kappa \mathrm{B}$; CREB, cyclic adenosine monophosphate response element binding protein.

reasons. First, TP $\alpha$ is believed to facilitate lung cancer growth, invasion and metastasis $[8,9,11-13]$. Second, the effects of both glycyrrhizin and cisplatin are related to the TxA2 pathway $[1,5,15,18]$. Third, the lung is a TxA2 enrichment organ $[8,14]$. Therefore, with the overexpression of TP $\alpha$, A549 cells can be facilitated to metastasize to the lung due to molecular predilection $[1,16]$.

In the present study, TxAS and PCNA were upregulated in tumor-bearing mice, which is not surprising because there is a positive feedback loop between TxAS and TP $\alpha$ in lung tumors [4]. In addition, PCNA is a well-known marker of tumor cell proliferation, and TxAS has been recently established as a promoter for tumor growth and metastasis [8, 12, 22]. We found that treatment of the mouse xenograft model with glycyrrhizin decreased expression of TxAS and PCNA, a finding that is in agreement with that in previous in vitro studies showing that glycyrrhizin and its hydrolysate 18ß-glycyrrhetinic acid can induce apoptosis and suppress the proliferation of lung cancer cells and that such an effect is, to some extent, 
TxAS dependent $[1,3,5]$. To support our results, there is a recent report demonstrating that the specific TxA2 inhibitor aspirin can prevent tumor metastasis and enhance TxA2 production in an animal model of colorectal cancer [23].

The anti-tumor effect of glycyrrhizin observed in the animal model might be expected to be of considerable clinical relevance. As we know, cisplatin has been employed as a firstline treatment for patients with lung tumors. However, the multiple organ-affected toxicity of this drug presents a major obstacle to attempt to improve the prognosis of patients with lung cancer [24]. Fortunately, this in vivo study found that glycyrrhizin can attenuate the organ-affected toxicity of cisplatin, as evidenced by the data where, compared with treatment with cisplatin alone, combined cisplatin with glycyrrhizin was more effective in recovering the body weight in tumor-bearing mice and rescuing animals from the damage to the liver and kidney caused by tumor bearing.

In addition to attenuating cisplatin toxicity, glycyrrhizin can sensitize A549 cells to cisplatin, which was demonstrated in our previous study [1]. As stated above, TxA2 has also been implicated in the resistance to chemotherapy [6]. It was reported that pharmacological inhibition of TxAS using the TxAS inhibitor furegrelate increased sensitivity to the chemotherapeutic agent cisplatin and paclitaxel in bladder cancer cells [15]. In addition, the pre-exposure of tumor cells to the TxA2-specific inhibitor aspirin lowers the concentration of cisplatin necessary to exert its cytotoxic action [25]. Coupled with the suppression effect of glycyrrhizin on TxAS, these observations indicate that the beneficial effects produced by the combination of cisplatin and glycyrrhizin can be somewhat attributable to blocking the TxA2 pathway. In support of this conclusion, it is now clear that the cytotoxic action of cisplatin is thought to be mediated, in part, through the TxA2 pathway $[18,26]$. Moreover, intriguingly, there is a report demonstrating that, due to the lack of suppression of TxA2, celecoxib enhanced the cytotoxic effect of cisplatin in chemo-resistant gastric cancer xenograft mouse models [18]. Collectively, the benefits of cisplatin combined with glycyrrhizin are excitingly promising, such as decreasing the toxicity and reducing the resistance to chemotherapy.

In summary, our studies presented evidence for the herbal drug glycyrrhizin to be a promising candidate for the treatment of lung adenocarcinoma in an in vivo mouse model by interacting with TxA2 abundance. Moreover, glycyrrhizin presented a profound effect on reversing the organ-affected toxicity of cisplatin. As proposed in Fig. 5D, the mechanism underlying glycyrrhizin action is possibly through suppressing the TxA2 pathway (a positive feedback loop between TxAS and TP $\alpha$ ).

There are several limitations of this study. Considering that the combination of platinum compounds and third-generation drugs, such as gemcitabine and vinorelbine, has been used as the first-line chemotherapy for non-small cell lung cancer, further work should consider more positive controls to match the clinical practice routinely used. Moreover, several crucial molecules involved in lung adenocarcinoma, such as epidermal growth factor receptor (EGFR) and anaplastic lymphoma kinase (ALK), should be considered to determine the effect of glycyrrhizin on signaling pathways. Furthermore, in future studies, it will be very interesting to analyze the form of glycyrrhizin that suppresses TxAS in mouse models using immunocompetent animals and syngeneic murine lung adenocarcinoma cell lines.

\section{Acknowledgements}

We sincerely thank Prof. Boudewijn Burgering for his comments and suggestions in conducting this study and writing this article.

This study was supported by the National Natural Science Foundation of China (No. No.81302799), the Science and Technology project of Guangdong Province (No. 2014A020221028 and No. 2016A020226041), as well as the Chinese Medical Science and Technology research funding from Guangdong Provincial Hospital of Chinese Medicine (No. YN2014ZH04 and No. YN2014PJ R202). 


\section{Cellular Physiology Cell Physiol Biochem 2017;41:1383-1392

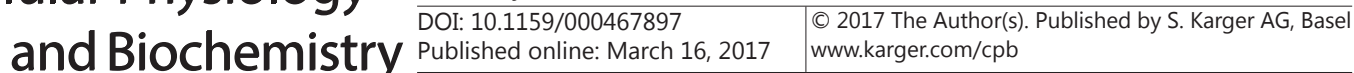 \\ Deng et al.: Glycyrrhizin Effects in a Lung Adenocarcinoma Model}

\section{Disclosure Statement}

The authors declare that they have no commercial or financial conflicts of interest.

\section{References}

1 Huang RY, Chu YL, Jiang ZB, Chen XM, Zhang X, Zeng X: Glycyrrhizin suppresses lung adenocarcinoma cell growth through inhibition of thromboxane synthase. Cell Physiol Biochem 2014;33:375-388.

-2 Peng F, Du Q, Peng C, Wang N, Tang H, Xie X, Shen J, Chen J: A Review: The Pharmacology of Isoliquiritigenin. Phytother Res 2015;29:969-977.

-3 Tang ZH, Li T, Tong YG, Chen XJ, Chen XP, Wang YT, Lu JJ: A Systematic Review of the Anticancer Properties of Compounds Isolated from Licorice (Gancao). Planta Med 2015;81:1670-1687.

-4 Xiong H, Xu Y, Tan G, Han Y, Tang Z, Xu W, Zeng F, Guo Q: Glycyrrhizin ameliorates imiquimod-induced psoriasis-like skin lesions in BALB/c mice and inhibits TNF-alpha-induced ICAM-1 expression via NFkappaB/MAPK in HaCaT cells. Cell Physiol Biochem 2015;35:1335-1346.

5 Huang RY, Chu YL, Huang QC, Chen XM, Jiang ZB, Zhang X, Zeng X: 18beta-Glycyrrhetinic acid suppresses cell proliferation through inhibiting thromboxane synthase in non-small cell lung cancer. PLoS One 2014;9:e93690.

6 Yang WS, Han NJ, Kim JJ, Lee MJ, Park SK: TNF-alpha Activates High-Mobility Group Box 1 - Toll-Like Receptor 4 Signaling Pathway in Human Aortic Endothelial Cells. Cell Physiol Biochem 2016;38:21392151.

7 Yang WS, Kim JS, Han NJ, Lee MJ, Park SK: Toll-like receptor 4/spleen tyrosine kinase complex in high glucose signal transduction of proximal tubular epithelial cells. Cell Physiol Biochem 2015;35:2309-2319.

-8 Ekambaram P, Lambiv W, Cazzolli R, Ashton AW, Honn KV: The thromboxane synthase and receptor signaling pathway in cancer: an emerging paradigm in cancer progression and metastasis. Cancer Metastasis Rev 2011;30:397-408.

9 Huang QC, Huang RY: The cyclooxygenase-2/thromboxane A2 pathway: a bridge from rheumatoid arthritis to lung cancer? Cancer Lett 2014;354:28-32.

10 Huang RY, Chen GG: Cigarette smoking, cyclooxygenase-2 pathway and cancer. Biochim Biophys Acta 2011;1815:158-169.

11 Huang RY, Li MY, Ng CS, Wan IY, Kong AW, Du J, Long X, Underwood MJ, Mok TS, Chen GG: Thromboxane A2 receptor alpha promotes tumor growth through an autoregulatory feedback pathway. J Mol Cell Biol 2013;5:380-390.

12 Huang RY, Li SS, Guo HZ, Huang Y, Zhang X, Li MY, Chen GG, Zeng X: Thromboxane A2 exerts promoting effects on cell proliferation through mediating cyclooxygenase- 2 signal in lung adenocarcinoma cells. J Cancer Res Clin Oncol 2014;140:375-386.

13 Li X, Tai HH: Thromboxane A2 receptor-mediated release of matrix metalloproteinase-1 (MMP-1) induces expression of monocyte chemoattractant protein-1 (MCP-1) by activation of protease-activated receptor 2 (PAR2) in A549 human lung adenocarcinoma cells. Mol Carcinog 2014;53:659-666.

14 Liu Y, Yang S, Li MY, Huang R, Ng CS, Wan IY, Long X, Wu J, Wu B, Du J, Mok TS, Underwood MJ, Chen GG: Tumorigenesis of smoking carcinogen 4-(methylnitrosamino)-1-(3-pyridyl)-1-butanone is related to its ability to stimulate thromboxane synthase and enhance stemness of non-small cell lung cancer stem cells. Cancer Lett 2016;370:198-206.

15 Moussa 0, Riker JM, Klein J, Fraig M, Halushka PV, Watson DK: Inhibition of thromboxane synthase activity modulates bladder cancer cell responses to chemotherapeutic agents. Oncogene 2008;27:55-62.

16 Wei J, Yan W, Li X, Ding Y, Tai HH: Thromboxane receptor alpha mediates tumor growth and angiogenesis via induction of vascular endothelial growth factor expression in human lung cancer cells. Lung Cancer 2010;69:26-32.

17 Huang RY, Li MY, Hsin MK, Underwood MJ, Ma LT, Mok TS, Warner TD, Chen GG: 4-Methylnitrosamino-13-pyridyl-1-butanone (NNK) promotes lung cancer cell survival by stimulating thromboxane A2 and its receptor. Oncogene 2011;30:106-116. 


\section{Cellular Physiology Cell Physiol Biochem 2017;41:1383-1392

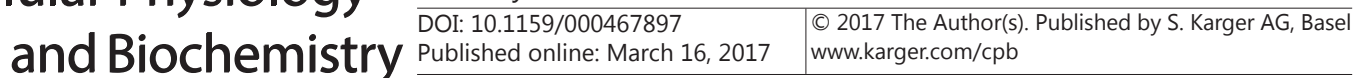 \\ Deng et al.: Glycyrrhizin Effects in a Lung Adenocarcinoma Model}

18 Xu HB, Shen FM, Lv QZ: Celecoxib enhanced the cytotoxic effect of cisplatin in chemo-resistant gastric cancer xenograft mouse models through a cyclooxygenase-2-dependent manner. Eur J Pharmacol 2016;776:1-8.

19 Dong X, Guan J, English JC, Flint J, Yee J, Evans K, Murray N, Macaulay C, Ng RT, Gout PW, Lam WL, Laskin J, Ling V, Lam S, Wang Y: Patient-derived first generation xenografts of non-small cell lung cancers: promising tools for predicting drug responses for personalized chemotherapy. Clin Cancer Res 2010;16:1442-1451.

-20 Nishimoto Y, Hisatsune A, Katsuki H, Miyata T, Yokomizo K, Isohama Y: Glycyrrhizin attenuates mucus production by inhibition of MUC5AC mRNA expression in vivo and in vitro. J Pharmacol Sci 2010;113:7683.

21 Sun L, Ma JT, Zhang SL, Zou HW, Han CB: Efficacy and safety of chemotherapy or tyrosine kinase inhibitors combined with bevacizumab versus chemotherapy or tyrosine kinase inhibitors alone in the treatment of non-small cell lung cancer: a systematic review and meta-analysis. Med Oncol 2015;32:473.

22 Wang MT, Honn KV, Nie D: Cyclooxygenases, prostanoids, and tumor progression. Cancer Metastasis Rev 2007;26:525-534.

23 Guillem-Llobat P, Dovizio M, Bruno A, Ricciotti E, Cufino V, Sacco A, Grande R, Alberti S, Arena V, Cirillo M, Patrono C, FitzGerald GA, Steinhilber D, Sgambato A, Patrignani P: Aspirin prevents colorectal cancer metastasis in mice by splitting the crosstalk between platelets and tumor cells. Oncotarget 2016;7:3246232477.

-24 Arrieta O, Michel Ortega RM, Villanueva-Rodriguez G, Serna-Thome MG, Flores-Estrada D, Diaz-Romero C, Rodriguez CM, Martinez L, Sanchez-Lara K: Association of nutritional status and serum albumin levels with development of toxicity in patients with advanced non-small cell lung cancer treated with paclitaxelcisplatin chemotherapy: a prospective study. BMC Cancer 2010;10:50.

25 Kumar A, Singh SM: Priming effect of aspirin for tumor cells to augment cytotoxic action of cisplatin against tumor cells: implication of altered constitution of tumor microenvironment, expression of cell cycle, apoptosis, and survival regulatory molecules. Mol Cell Biochem 2012;371:43-54.

-26 Dorner GT, Pehamberger H, Kornek G, Steger G, Muller M, Wolzt M, Kletter K, James P, Breiteneder H, Eichler HG, Blochl-Daum B: Cisplatin-induced renal effects and thromboxane A2 receptor blockade. Eur J Clin Invest 1997;27:836-839. 